\title{
Restoration of Blurred Binary Images Using Discrete Tomography
}

\author{
Jozsef Nemeth $^{1}$ and Peter Balazs ${ }^{2}$ \\ ${ }^{1}$ Department of Computer Algorithms and Artificial Intelligence, \\ ${ }^{2}$ Department of Image Processing and Computer Graphics, \\ University of Szeged, H-6701 Szeged, PO. Box 652., Hungary, Fax: +36 62 546-397 \\ \{nemjozs, pbalazs\}@inf.u-szeged.hu
}

\begin{abstract}
Enhancement of degraded images of binary shapes is an important task in many image processing applications, e.g. to provide appropriate image quality for optical character recognition. Although many image restoration methods can be found in the literature, most of them are developed for grayscale images. In this paper we propose a novel binary image restoration algorithm. As a first step, it restores the projections of the shape using 1-dimensional deconvolution, then reconstructs the image from these projections using a discrete tomography technique. The method does not require any parameter setting or prior knowledge like an estimation of the signal-to-noise ratio. Numerical experiments on a synthetic dataset show that the proposed algorithm is robust to the level of the noise. The efficiency of the method has also been demonstrated on real out-of-focus alphanumeric images.
\end{abstract}

\section{Introduction}

Restoration of distorted images is a key step in many applications. Degradations usually come in many forms such as motion blur, camera misfocus and noise. Misfocus and motion blur can be described by so called blurring functions (filters) while noise usually follows a specific distribution as a good approximation.

There are many methods in the literature for digital image enhancement including simple ones and more sophisticated algorithms. Unsharp masking [12] is one of the widely used tools to enhance image contrast. This basically photographic technique is used since the first half of the 20th century to increase the sharpness of images. Its digital version is used in many image processing softwares. Inverse filtering (or deconvolution) of images is another technique for recovering an image that is blurred by a known low-pass filter. It tries approximately inverting the process that caused an image to be blurred. Researchers have been studying deconvolution methods for several decades, and have approached the problem from different directions.

Basically, deconvolution is very sensitive to noise. This problem can be handled using the Wiener filter [16] which can be used when the point-spread-function (PSF, the filter which was used to blur the image) and the signal-to-noise ratio are known. Blind deconvolution [6] is a technique for recovering an image that was blurred using an unknown PSF. This iterative approach improves the estimation of the PSF and the 
scene in each iteration, thus the convergence depends on how accurate is the initial estimation of the PSF.

The restoration of bi-level images like document images has been addressed by several researchers (see [4], [5], [7] and [14]). Most of these methods trace back the problem to the solution of a system of equations which then solved by iterative optimization. Although these techniques are intensely studied, they are still time-consuming and require good parameter settings which makes the usage of them difficult in practice. The size of the point spread function is usually also limited.

In [13] Sharif et al. proposed a discrete tomography based binary deconvolution method. The size of the PSF was restricted to $3 \times 3$ and the restoration of the projections was handled as a simple linear inverse problem. Moreover, the method applied the Ryser's algorithm [11] requiring a further time-consuming process to resolve the switching component ambiguities.

In this paper we propose an efficient Tikhonov-regularization [15] based method to reconstruct the projections of the original binary shapes. The main advantage of this approach is that the best value of the regularization parameter can be automatically set using the L-curve method [3]. In the second step the binary shapes are reconstructed using efficient network-flow discrete tomography algorithm [1]. The efficiency of the method has been examined on synthetic as well as on real images. The performance of the proposed method has also been compared to a well-known, widely-used method.

This paper is organized as follows. Section 2 describes the proposed method. In Section 2.1 the deconvolution of the projections is discussed, while in Section 2.2 the discrete tomography reconstruction method is described. Section 3 presents the experimental results.

\section{The method}

The goal of image restoration is to reconstruct an original image $f \in \mathbb{R}^{u \times v}$ from its degraded observation $g \in \mathbb{R}^{u \times v}$ which is blurred and noisy. The degradation process (as usually) modelled as the convolution of the original image with the PSF and the addition of some noise:

$$
g=h * * f+n,
$$

where $h \in \mathbb{R}^{p \times q}$ is the point spread function, $* *$ denotes the 2-dimensional convolution operator and $n \in \mathbb{R}^{u \times v}$ is the additive noise. In this paper we assume that the PSF is known. Our goal is to restore binary images, such as $b \in\{0,1\}^{u \times v}$. However, in the case of real images the scaling of the intensity values can be arbitrary. Therefore we introduce the unknown scale factor $s \in \mathbb{R}$ and

$$
f=s b
$$

in the degradation model Eq. (1).

Let denote by $\check{f}^{\theta}$ the projection vector of any image $f$ along any directional angle $\theta$, i.e. $\check{f}^{\theta}$ is the discrete approximation of the well-known Radon transform [2] in direction $\theta$ (we simply write $\check{f}$ when the actual direction is not important). Here we take advantage of the following property of the Radon transform: the result of the Radon transform 


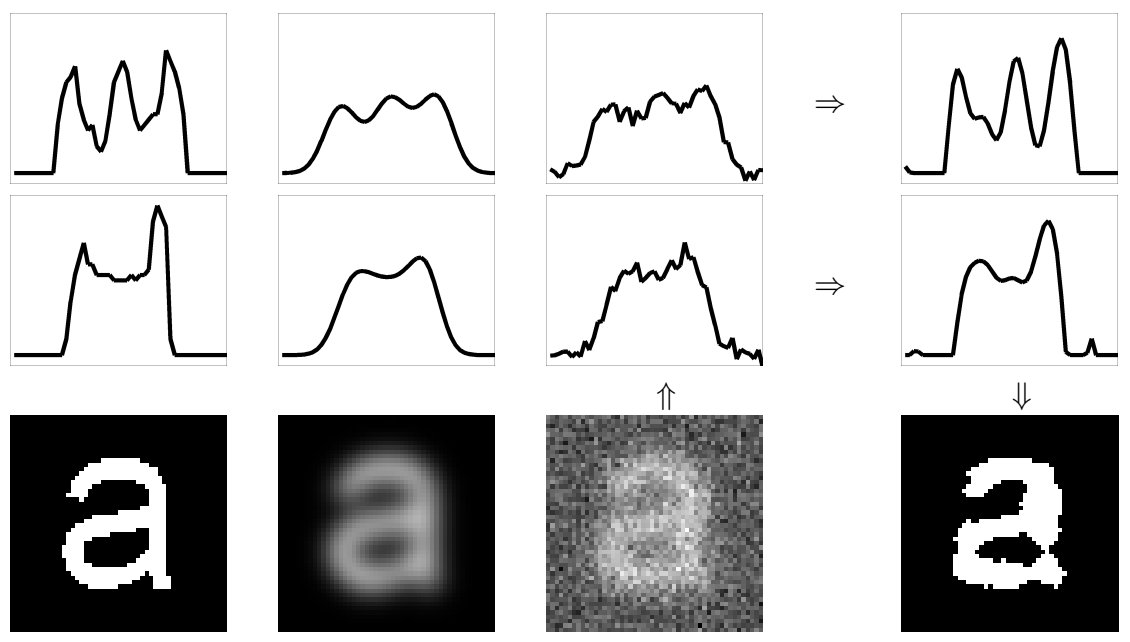

Fig. 1. The degradation model and the basic idea of the proposed method. First three columns: the original binary image, the blurred image and the blurry and noisy image and their horizontal (top) and vertical (middle) projections. Fourth column: the method restores the projections of the binary image from the projections of the degraded image then reconstructs the binary shape.

of a two dimensional convolution is the same as the one dimensional convolution of the Radon transformed functions, i.e. if $g=h * * f$ then $\check{g}=\check{h} * \check{f}$, where $*$ denotes the 1-dimensional convolution operator. A similar relation is true for the addition. Thus the relationship between the projections of the original and the degraded images can be expressed as

$$
\check{g}=\check{h} * \check{f}+\check{n} .
$$

For a direction set $\Omega=\left\{\theta_{i} \mid i=1, \ldots, k\right\}$ the proposed method first estimates each projection $\check{f}^{\theta_{i}}$ of the unknown original image from the projections $\check{g}^{\theta_{i}}$ of the degraded image and then as a second step reconstructs $f$ from its estimated projections (see Figure 1).

\subsection{Deconvolution of the projections}

In this section we introduce a Tikhonov-regularization [15] based method for the deconvolution of the projections. Convolution is a linear operation, thus Eq. (3) can be written as

$$
\check{g}=H \check{f}+\check{n},
$$

where the $H$ matrix represents the convolution operation with the filter $\breve{h}$. Since $\check{n}$ is unknown, this system of linear equations is ill-posed and it requires regularization which penalizes solutions of large norm. The standard version of the Tikhonov regularization takes the form

$$
\check{f}_{\lambda}=\underset{\check{f}}{\arg \min }\|H \check{f}-\check{g}\|_{2}^{2}+\lambda^{2}\|\check{f}\|_{2}^{2},
$$




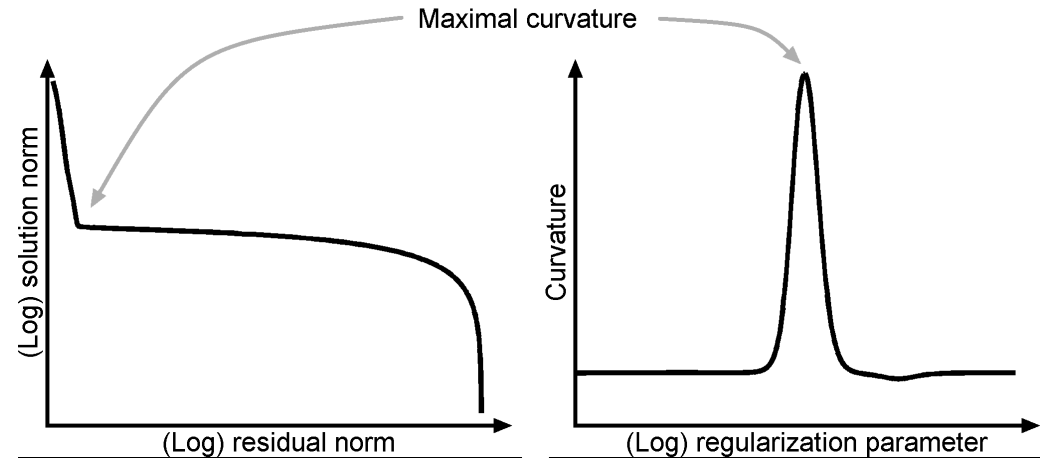

Fig. 2. A typical L-curve and its curvature. The best trade-off between the norm of the residual and the norm of the solution is assigned by the maximal curvature value.

where $\lambda$ is a positive constant, the regularization parameter, that controls the smoothness of the solution. As $\lambda$ increases the norm of the residual $\left\|H \check{f}_{\lambda}-\check{g}\right\|_{2}^{2}$ also increases (i.e. $\check{f}_{\lambda}$ becomes less accurate), while the norm of the solution $\left\|\check{f}_{\lambda}\right\|_{2}^{2}$ decreases (i.e. $\check{f}_{\lambda}$ becomes smoother). An explicit solution for a given value of $\lambda$ is given by

$$
\check{f}_{\lambda}=\left(H^{T} H+\lambda^{2} I\right)^{-1} H^{T} \check{g},
$$

where $I$ denotes the identity matrix. To determine a suitable value of the regularization parameter $\lambda$ we used the so-called L-curve method [3]. The L-curve is a log-log plot of the norm of the residual and the norm of the solution for different regularization parameters:

$$
\mathcal{L}=\left\{\left(\log _{2}\left\|H \check{f}_{\lambda}-\check{g}\right\|_{2}^{2}, \log _{2}\left\|\check{f}_{\lambda}\right\|_{2}^{2}\right), \lambda \geq 0\right\} .
$$

To obtain an optimal trade-off between the two values, the L-curve method proposes to choose $\lambda^{*}$ which maximizes the curvature $\kappa_{\lambda}$ of the curve $\mathcal{L}$ (see Figure 2). Although the L-curve method requires for a large set of different $\lambda$ values to evaluate Eq. (6), it is still computationally efficient approach.

Since the elements of the projection vectors are non-negative we are interested in a solution of Eq. (4) such that $\check{f}>0$. Unfortunately there is no simple explicit solution for this problem, but a typical iterative approach can be applied. For that purpose, we denoted by $\check{f}^{+}$the version of the vector $\check{f}$ in which all negative values were set to 0 . Let $\check{f}_{0}=\check{f}_{\lambda^{*}}$ the initial estimation. In each iteration step the method estimates $\check{f}_{i}$ that approximates $\check{f}_{i-1}^{+}$(see Figure 3). This can be formulated by the following minimization problem:

$$
\check{f}_{i}=\underset{\check{f}}{\arg \min }\|H \check{f}-\check{g}\|_{2}^{2}+\lambda^{* 2}\|\check{f}\|_{2}^{2}+\left\|\check{f}-\check{f}_{i-1}^{+}\right\|_{2}^{2}
$$

which introduces an additional term to Eq. (5) that prefers similarity to the non-negative version of the previous estimation. This form of the generalized Tikhonov regularization has an explicit solution:

$$
\check{f}_{i}=\left(H^{T} H+\left(1+\lambda^{* 2}\right) I\right)^{-1}\left(H^{T} \check{g}+\check{f}_{i-1}^{+}\right) .
$$




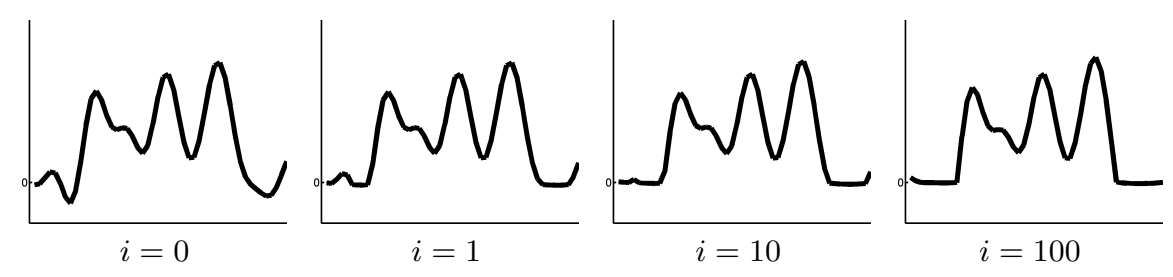

Fig. 3. The evolution of the estimation of the projection vector during the iteration.

The iteration ends if $\left\|\check{f}_{i}-\check{f}_{i-1}\right\|_{2}^{2}<\epsilon\left\|\check{f}_{i-1}\right\|_{2}^{2}$ for an appropriately small $\epsilon>0$. The resulting vector $\check{f}=\check{f}_{i}^{+}$will be the estimation of the projection vector. The pseudo code of this algorithm can be found in Algorithm 1. Furthermore the residual $\|H \check{f}-\check{g}\|_{2}^{2}$ approximates $\check{n}$ and thus the variance $\sigma_{n}^{2}$ of the additive noise $n$ can be easily estimated.

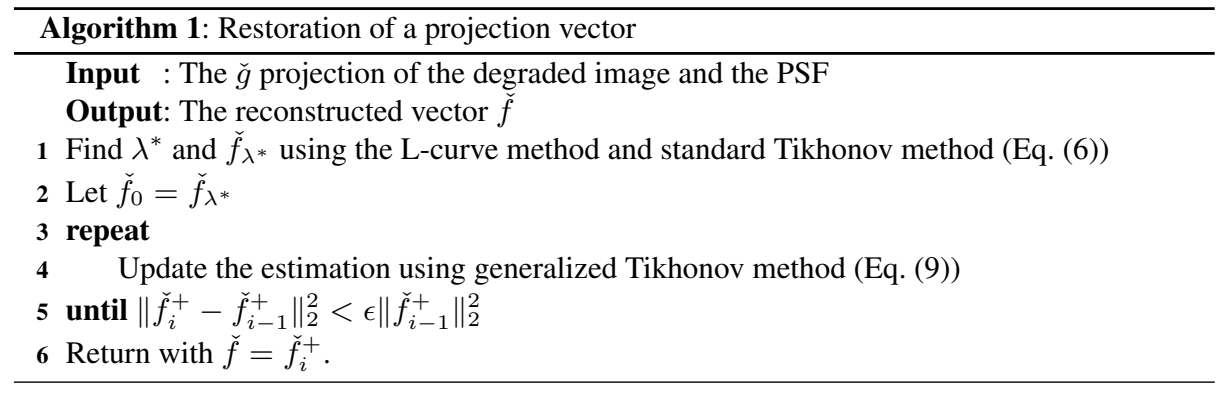

\subsection{Binary image reconstruction}

In this section we propose a method to reconstruct the binary image from the vectors $\check{f}^{\theta_{i}}$ using a standard discrete tomography technique. The reconstruction requires the $\breve{b}^{\theta_{i}}=\check{f}^{\theta_{i}} / s$ binary projections. Unfortunately the scale factor $s$ can not be determined explicitly but the upper and lower bounds of its possible values can be easily estimated. For that purpose, consider the maximal scaled projection value $M=\max _{\theta_{i}}\left\{\max \left\{\check{f}^{\theta_{i}}\right\}\right\}$ and let $D$ be the length of the segment of the image that the projection ray corresponding to $M$ intersects. It can be assumed that $1 \leq M / s \leq D$ so we can define a set of the possible values of the scale factor $s$ as follows:

$$
S=\{k / M, k=1, \ldots,\lfloor D\rfloor\} .
$$

For each $s \in S$ we obtain the vectors $\check{b}^{\theta_{i}}=\left[\check{f}^{\theta_{i}} / s\right]$ as estimations of the projections of the unknown binary image $b$.

In this paper we consider the reconstruction from the vertical and horizontal projection vectors $\breve{b}^{0}$ and $\breve{b}^{\pi / 2}$. The discrete tomography technique described in in this section 


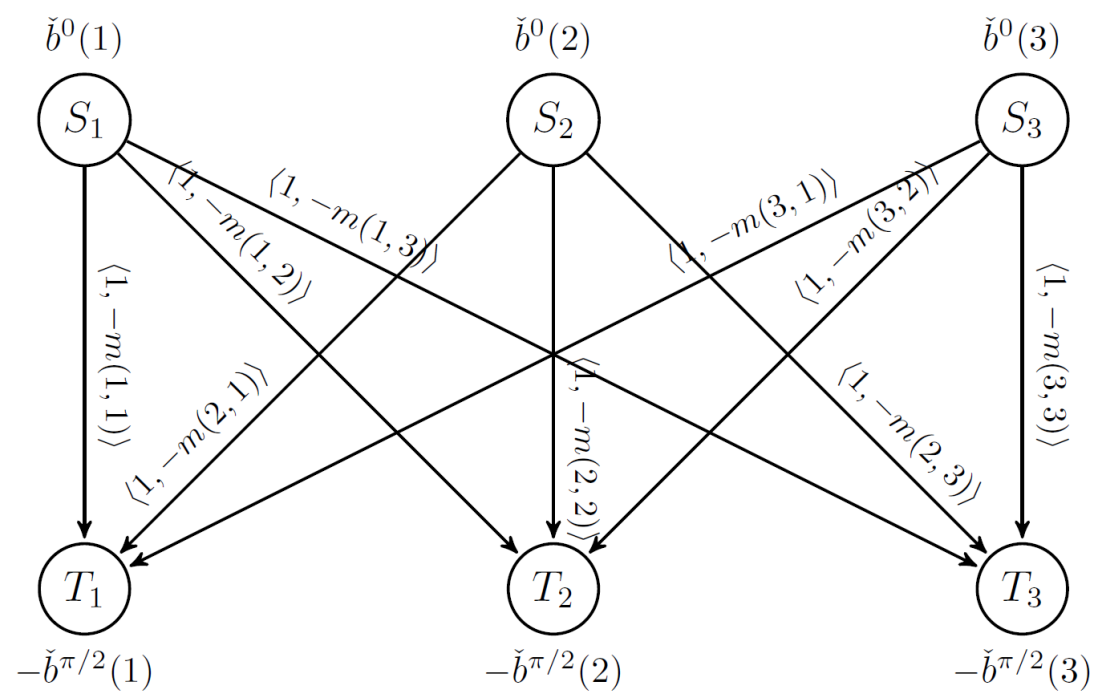

Fig. 4. Associated network for a $3 \times 3$ case. The supply and demand values for the source nodes $S_{i}$ and the sink nodes $T_{j}$ are given by the vectors $\breve{b}^{0}$ and $\breve{b}^{\pi / 2}$ respectively. Each of the edges has a flow capacity equal to 1 while the flow costs are given by the model image $m \in \mathbb{R}^{3 \times 3}$.

requires that these two vectors have equal sum. However, in practice this is not guaranteed since these vectors are obtained by rounding. To equalize the two vectors we uniformly decreased the elements of the vector of the largest sum. We decreased by 1 the highest elements of the same number as the difference between the two vectors.

It is well known, that usually two projections are not enough to reconstruct binary images, i.e. , there can be many binary images that have the same projections while in some other cases there is no binary image that satisfies the projections. We define the tomographic equivalence class:

$$
U=U\left(\check{b}^{0}, \check{b}^{\pi / 2}\right)=\left\{z \in\{0,1\}^{u \times v}: \check{z}^{0}=\check{b}^{0}, \check{z}^{\pi / 2}=\check{b}^{\pi / 2}\right\} .
$$

Although in general case $|U|>1$, we are interested in a solution which is the most similar to the input image $g$. Fortunately finding such a solution is quite straightforward. For that purpose a model image $m$ has been created from the input image $g$ by removing noise of variance $\sigma_{n}^{2}$ (which has been estimated after the estimation of the projections, see Section 2.1). The expectation that the solution $z_{s}$ should be similar to the model image means that on those $(x, y)$ positions where $z(x, y)=1$ it is most likely that the model image has high grayscale values. This can be formulated by the following minimization problem:

$$
z_{s}=\underset{z \in U}{\arg \min }\left(-\sum_{x, y} z(x, y) m(x, y)\right) .
$$

This way the reconstruction problem can be traced back to the minimum cost maximal flow (MCMF) problem [1]. In this network the supply and demand nodes are repre- 


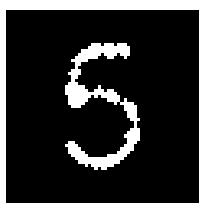

$s=0.64$

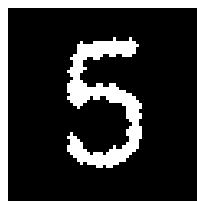

$s=0.92$

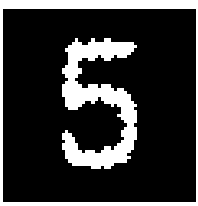

$s=1.07$

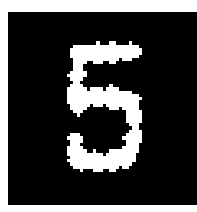

$s=1.21$

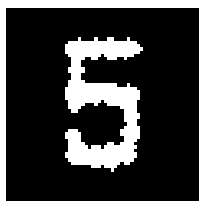

$s=1.32$

Fig. 5. Reconstruction results for different $s \in S$ scale values.

senting the projection vectors while the edges are representing the image pixels (see Figure 4). The edge $S_{x} \rightarrow T_{y}$ corresponding to the $(x, y)$ pixel has a flow capacity equal to 1 and a flow cost equal to $-m(x, y)$. The minimum cost maximal flow can be found in polynomial time [8] and it determines the solution $z_{s}$ of the discrete tomography problem. The pixel $z_{s}(x, y)$ gets a value of 1 , if and only if the flow passes through the edge $S_{x} \rightarrow T_{y}$. As it has been noted previously in some cases the projections can not be satisfied, i.e. , $U$ is empty. In such cases we simply discard the current scale factor and the corresponding projections. We also note that the original input image $g$ could also be used as model image, however the results would be somewhat more noisy.

For different scale factors $s \in S$ the method finds different binary solutions $z_{s}$ (see Figure 5). To choose an optimal solution $z$, the method compares each $z_{s}$ to the input image $g$ in least-squares sense

$$
z=\underset{z_{s}}{\arg \min }\left\|s\left(h * * z_{s}\right)-g\right\|^{2} .
$$

It should be noted that theoretically it is not impossible that $|U|=0$ for each scale factor $s \in S$. In this case the proposed method could not provide any solution. However in practice we found that $U$ containes at least one solution for most of $s \in S$.

The pseudo code of the algorithm can be found in Algorithm 2. As for the time complexity of the overall algorithm, the reconstruction of the projections require a matrix invertion in each iteration step which has a polynomial time consumption while the reconstruction of the binary shapes for each scale factor $s \in S$ using the MCMF approach has also polynomial time complexity. Hence the overall method is computationally efficient.

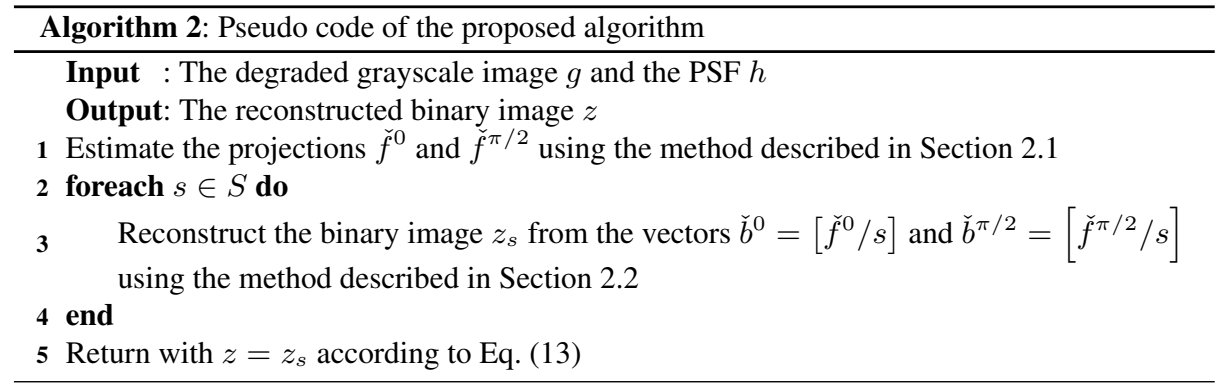


Richardson-Lucy method

$\sigma \quad 5 \mathrm{~dB} \quad 2.5 \mathrm{~dB} \quad 0 \mathrm{~dB} \quad-2.5 \mathrm{~dB} \quad-5 \mathrm{~dB}$

$\begin{array}{llllll}1.0 & 9.93 & 12.66 & 15.94 & 21.78 & 30.58\end{array}$

$\begin{array}{llllll}2.0 & 12.01 & 14.57 & 17.80 & 23.99 & 31.21\end{array}$

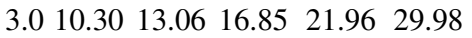

$\begin{array}{llllll}4.0 & 14.47 & 16.98 & 20.30 & 26.44 & 35.26\end{array}$

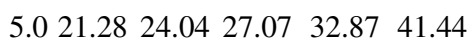

Proposed method

$\sigma \quad 5 \mathrm{~dB} \quad 2.5 \mathrm{~dB} \quad 0 \mathrm{~dB} \quad-2.5 \mathrm{~dB} \quad-5 \mathrm{~dB}$

$\begin{array}{llllll}1.0 & 10.83 & \mathbf{1 2 . 3 1} \mathbf{1 4 . 4 4} \mathbf{1 8 . 8 8} \mathbf{2 4 . 1 5}\end{array}$

$2.014 .67 \quad 15.69 \mathbf{1 7 . 1 9} \mathbf{2 0 . 8 0} \mathbf{2 2 . 6 1}$

$3.017 .41 \quad 18.3419 .94 \mathbf{2 1 . 6 6} \mathbf{2 4 . 5 8}$

$\begin{array}{llllll}4.0 & 21.53 & 23.38 & 25.17 & 27.53 & \mathbf{2 9 . 4 4}\end{array}$

$\begin{array}{lllll}5.0 & 26.47 & 28.43 & 30.90 & \mathbf{3 2 . 5 0} \mathbf{3 5 . 7 5}\end{array}$

Table 1. Test results on the synthetic dataset of the Richardson-Lucy method and the proposed method. The average $\delta$ measures on 62 alphanumeric characters for different levels of blur and SNR are shown. The cases when the proposed method provided better results are depicted in bold.

\section{Experiments and comparison}

To examine the performance of the method we created a synthetic dataset of images of 62 alphanumeric characters (of size $59 \times 59$ pixels) and their 1550 degraded versions. Each image was blurred by Gaussian filters with standard deviations $\sigma=0,1, \ldots, 5$. White noises of different levels was added to each blurred image to implement signalto-noise ratios covering values of $5,2.5,0,-2.5,-5 \mathrm{~dB}$. The reconstruction results were evaluated using the following normalized symmetric difference

$$
\delta=\frac{|B \triangle Z|}{|B|+|Z|} \cdot 100 \%
$$

where $B$ and $Z$ denote the set of the foreground pixels of the original image $b$ and the reconstructed image $z$ respectively.

For comparison we have chosen the Richardson-Lucy algorithm [10] because this method also assumes that the PSF is known and does not require an estimation of the strength of the noise. On the other hand this method is developed for grayscale images thus thresholding is required. The Otsu [9] method has been found as the best choice for this task. To improve the results of both methods, small isolated pixel groups has been removed from the images by the following way. First, a morphological closing has been applied to connect regions and isolated pixels that are close to each other. Then 8connected pixel groups containing at most a certain number of pixels has been removed. Finally, a logical AND operation with the original shape has been applied to undo the morphological closing. It has to be noted that the Richardson-Lucy method produced much more of such artifacts. The summary of the test results is shown in Table 3. It can be seen that the proposed method provided better results in low SNR cases. This is due to the L-curve based estimation of the regularization parameter which implicitly gives a robust estimation of the level of the noise. On the other hand, in higher SNR cases the 2-dimensional deconvolution methods (like the Richardson-Lucy algorithm) can provide more accurate restorations.

The Richardson-Lucy algorithm requires a couple of Fourier transforms in each iteration step, thus for fixed number of iterations, its time complexity is $\mathcal{O}(u v \log (u v))$. 


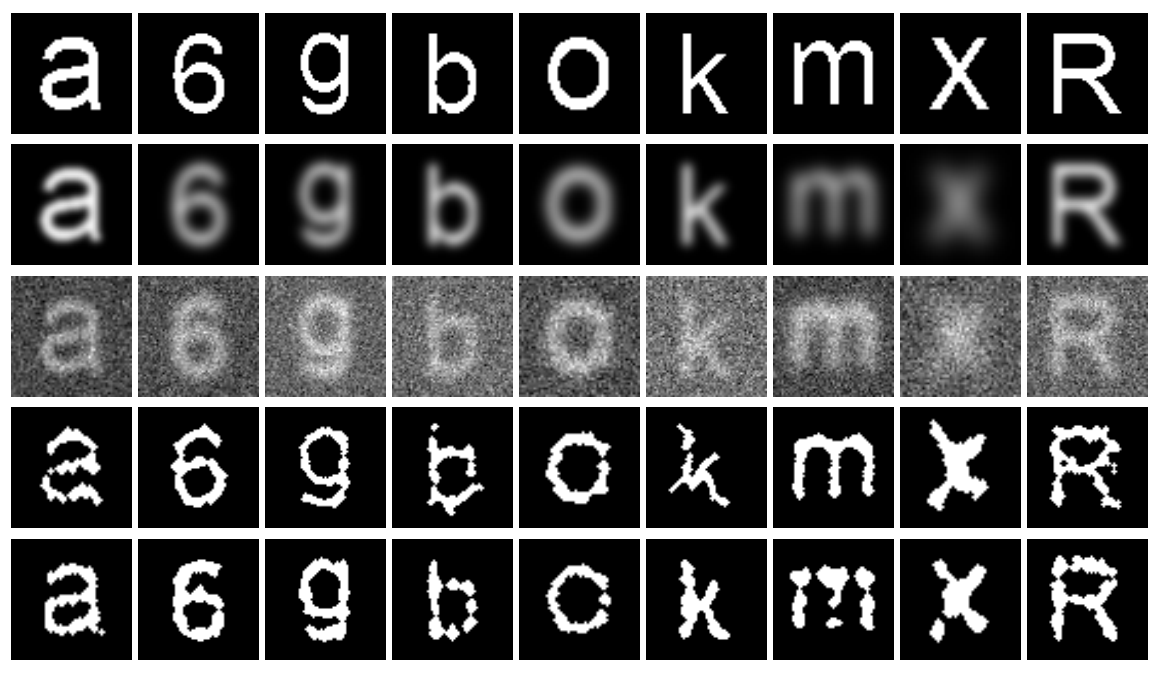

Fig. 6. Example reconstruction results on synthetic images. First three rows: The original binary shapes, the blurred images, and the blurred and noisy version (from top to middle). Fourth and fifth rows: The results of the Richardson-Lucy method and the proposed method respectively.

As we discussed in the previous section, the proposed method has a polynomial time complexity. We implemented our method in Mat lab, but the network-flow algorithm was written in C. This inefficient mixed solution resulted longer running times (an average of $\approx 5 \mathrm{sec}$.), while the Richardson-Lucy algorithm was very fast (below $0.1 \mathrm{sec}$.). However a more efficient implementation of our method is feasible.

\subsection{Real images taken with fixed-focus cameras}

Nowadays optical character recognition (OCR) is one of the most important functionalities of mobile devices. Nevertheless many modern devices are equipped with fixedfocus or full-focus (Extended Depth of Field - EDoF) lens. While these cameras in many cases provide better image quality than many auto-focus cameras, they usually can not be used for OCR because the images of A4 pages or business cards taken from a distance about $20 \mathrm{~cm}$ are out-of-focus. Therefore the enhancement of such images is an interesting task. We extracted a set of letters from images of A4 papers taken with an EDoF camera. Gaussian filters has been used as PSFs and their variances has been determined empirically. The results of the Richardson-Lucy method and the proposed method are shown in Figure 7. It can be observed that the proposed method provided in same cases better results, thus it can be regarded as a promising alternative. We note that it is not clear that Gaussian filter approximates the best the out-of-focus effect. Disk shaped low-pass filters has also been tested but while the proposed method performed similarly, the Richardson-Lucy method provided much weaker results. 


\section{$\mathbf{s}$ nVO 030

Fig. 7. Example results on letters extracted from out-of-focus document images taken with EDoF camera. The original images are shown in the first row. The reconstruction results of the Richardson-Lucy method can be found in the second row and the results of the proposed method are in the third row.

\section{Conclusion}

A novel binary image reconstruction approach has been proposed. The method first restores the projections of the shape using 1-dimensional deconvolution then reconstructs the binary image using efficient discrete tomography technique. The main advantage of the method is that it does not require parameter setting or prior knowledge like an estimation of the signal-to-noise ratio. The optimal trade-off between the residual and the accuracy is determined automatically. Numerical experiments on synthetic images showed that the method is robust to the level of the noise and can outperform a widelyused method in low SNR cases. The efficiency of the method has also been demonstrated on real alphanumeric images taken with fixed-focus camera.

\section{Acknowledgements}

This research was supported by the OTKA PD100950 project of the National Scientific Research Fund and by the European Union and the State of Hungary, co-financed by the European Social Fund in the framework of TÁMOP 4.2.4. A/2-11-1-2012-0001 'National Excellence Program'.

\section{References}

1. Batenburg, K.: Network flow algorithms for discrete tomography. In: Herman, G., Kuba, A. (eds.) Advances in Discrete Tomography and Its Applications, pp. 175-205. Applied and Numerical Harmonic Analysis, Birkhäuser Boston (2007)

2. Deans, S.R.: The Radon transform and some of its applications. A Wiley-Interscience publication, Wiley (1983)

3. Hansen, P.C., O'Leary, D.P.: The use of the L-curve in the regularization of discrete ill-posed problems. SIAM Journal on Scientific Computing 14, 1487-1503 (1993)

4. Kim, J., Jang, S.: High order statistics based blind deconvolution of bi-level images with unknown intensity values. Optics Express 18(12), 12872-12889 (June 2010)

5. Lam, E.Y.: Blind bi-level image restoration with iterated quadratic programming. IEEE Transactions on Circuits and Systems Part 2: Express Briefs 54(1), 52-56 (2007)

6. Lam, E.Y., Goodman, J.W.: Iterative statistical approach to blind image deconvolution. Journal of the Optical Society of America A 17(7), 1177-1184 (2000) 
7. Li, T.H., Lii, K.S.: Deblurring two-tone images by a joint estimation approach using higherorder statistics. In: Proc. of IEEE SP Workshop on Higher-Order Statistics. pp. 108-111 (1997)

8. Orlin, J.B.: A polynomial time primal network simplex algorithm for minimum cost flows. In: Proc. of the seventh annual ACM-SIAM symposium on Discrete algorithms. pp. 474481. SODA '96, SIAM, Philadelphia, PA, USA (1996)

9. Otsu, N.: A threshold selection method from gray-level histograms. IEEE Transactions on Systems, Man and Cybernetics 9(1), 62-66 (Jan 1979)

10. Richardson, W.H.: Bayesian-based iterative method of image restoration. J. Opt. Soc. Am. 62(1), 55-59 (Jan 1972)

11. Ryser, H.: Combinatorial properties of matrices of zeros and ones. In: Classic Papers in Combinatorics, pp. 269-275. Modern Birkhäuser Classics, Birkhäuser Boston (1987)

12. Schalkoff, R.J.: Digital Image Processing and Computer Vision. John Wiley \& Sons, New York, NY, USA (1989)

13. Sharif, B., Sharif, B.: Discrete tomography in discrete deconvolution: Deconvolution of binary images using ryser's algorithm. Electronic Notes in Discrete Mathematics 20, 555-571 (July 2005)

14. Shen, Y., Lam, E.Y., Wong, N.: Binary image restoration by positive semidefinite programming. Optics Letters 32(2), 121-123 (2007)

15. Tikhonov, A., Arsenin, V.: Solutions of ill-posed problems. Scripta series in mathematics, Winston (1977)

16. Wiener, N.: Extrapolation, Interpolation, and Smoothing of Stationary Time Series with Engineering Applications. John Wiley \& Sons, New York, NY, USA (1949) 